\title{
Reactivation capacity by latency-reversing agents ex vivo correlates with the size of the HIV-1 reservoir
}

\author{
Gilles Darcis ${ }^{\mathrm{a}, \mathrm{b}}$, Sophie Bouchat ${ }^{\mathrm{a}, *}$, Anna Kula ${ }^{\mathrm{a}, *}$, \\ Benoit Van Driessche ${ }^{a}$, Nadège Delacourt ${ }^{a}$, Caroline Vanhulle ${ }^{a}$, \\ Véronique Avettand-Fenoel ${ }^{c}$, Stéphane De Wit ${ }^{d}$, Olivier Rohr ${ }^{e, f}$, \\ Christine Rouzioux ${ }^{c}$ and Carine Van Lint ${ }^{a}$
}

\begin{abstract}
Objective: HIV-1 reservoirs are the major hurdle to virus clearance in combination antiretroviral therapy (cART)-treated patients. An approach to eradicating HIV-1 involves reversing latency in CART-treated patients to make latent cells visible to the host immune system. Stimulation of patient cell cultures with latency-reversing agents (LRAs) ex vivo results in heterogeneous responses among HIV-infected patients. Determinants of this heterogeneity are unknown and consequently important to determine.
\end{abstract}

Design and methods: Here, we grouped and retrospectively analyzed the data from our two recent HIV-1 reactivation studies to investigate the role of the HIV-1 reservoir size in the reactivation capacity by LRAs in ex vivo cultures of $\mathrm{CD}^{+}$-depleted peripheral blood mononuclear cells (PBMCs) isolated from 54 cART-treated patients and of resting $\mathrm{CD}^{+}{ }^{+} \mathrm{T}$ cells isolated from 30 cART-treated patients.

Results: Our results established a statistically relevant positive correlation between the HIV-1 reservoir size measured by total cell-associated HIV-1 DNA and the frequency of positive HIV-1 recovery measurements in response to various LRAs in ex vivo cultures of cells isolated from cART-treated $\mathrm{HIV}^{+}$aviremic patients. HIV-1 reservoir size also correlated with the extracellular HIV-1 RNA median level measured in supernatants of cell cultures following LRA treatments. However, we identified $\mathrm{HIV}^{+}$patients whose positive measurements frequency and median level of extracellular HIV-1 RNA deviated from linearity relative to their corresponding HIV reservoir size.

Conclusion: We demonstrated that the reservoir size is one predictive marker of LRA effectiveness but this parameter alone is not sufficient. The identification of other predictive markers is necessary to predict the success of HIV anti-latency approaches. Copyright (๑ 2017 Wolters Kluwer Health, Inc. All rights reserved.

AIDS 2017, 31:181-189

Keywords: anti-HIV strategy, HIV reactivation, HIV reservoir size, latencyreversing agent, predictive markers of latency-reversing agent effectiveness

\footnotetext{
a Service of Molecular Virology, Département de Biologie Moléculaire (DBM), Université Libre de Bruxelles (ULB), Gosselies, ${ }^{\mathrm{b}}$ Service des Maladies Infectieuses, Université de Liège, Centre Hospitalier Universitaire (CHU) de Liège, Domaine Universitaire du Sart-Tilman, Liège, Belgium, ' Service de Virologie, Université Paris-Descartes, AP-HP, Hôpital Necker-Enfants Malades, Paris, France, ${ }^{d}$ Service des Maladies Infectieuses, CHU St-Pierre, ULB, Bruxelles, Belgium, ${ }^{\mathrm{e}}$ Institut de Parasitologie et de Pathologie Tropicale, EA7292, and Institut Universitaire de Technologie Louis Pasteur de Schiltigheim, University of Strasbourg, Strasbourg, France.

Correspondence to Carine Van Lint, Service of Molecular Virology, Département de Biologie Moléculaire (DBM), Université Libre de Bruxelles (ULB), Rue des Professeurs Jeener et Brachet 12, 6041 Gosselies, Belgium.

E-mail: cvlint@ulb.ac.be

* Sophie Bouchat and Anna Kula contributed equally to the article.

Received: 19 May 2016; revised: 2 September 2016; accepted: 19 September 2016.
}

DOI:10.1097/QAD.0000000000001290

ISSN 0269-9370 Copyright (c) 2017 Wolters Kluwer Health, Inc. All rights reserved. 


\section{Introduction}

HIV-1 reservoirs impede virus clearance in patients under combination antiretroviral therapy (cART) [1]. Activation of HIV-1 expression from latent reservoirs is a part of proposed strategies that may potentially lead to a decrease in the size of the reservoirs [2]. Cells harboring induced proviruses could then be lysed by viral cytopathic effects or host cytolytic effector mechanisms, whereas new rounds of infection are blocked by cART [3]. Latency-reversing agents (LRAs) have been identified through different mechanism-based approaches and include histone deacetylase inhibitors (HDACis), DNA methyltransferase inhibitors, protein kinase $\mathrm{C}$ (PKC) agonists and bromodomain and extra-terminal (BET) inhibitors (BETi) [4]. We and others have demonstrated the HIV-1 reactivation potential of combined treatments with different classes of LRAs ([5-8], reviewed in [4]). These studies highlight a high diversity of responses among ex-vivo patient cell cultures in terms of reactivation capacity following treatment with LRAs. The understanding of the determinants involved in these patient-specific reactivation variations would be a key progress made toward the identification of means to reverse latency. Notably, little is known about the HIV-1 reservoir size role in the reactivation of patient cell cultures by LRAs. In this report, we analyzed patient data from two of our most recent reactivation studies to reach a number of patients sufficient for an accurate and statistically relevant analysis of the results [8,9]. In the first study, we have shown that combined treatments of PKC agonists with compounds releasing active positive transcription factor $\mathrm{b}$ (P-TEFb) synergistically reactivate HIV-1 in ex-vivo cultures of primary cells isolated from blood of cART-treated $\mathrm{HIV}^{+}$aviremic patients [8]. In our second recently published study performed with the same postintegration latency models, we have demonstrated that combined treatments of the nucleoside analogue DNA methylation inhibitor 5-aza-2'-deoxycytidine (5-AzadC (Sigma, Belgium), marketed as Dacogen) with clinically tolerable HDACis synergistically reactivates HIV-1 from latency [9]. Importantly, this synergy between 5-AzadC and HDACis was stronger when a sequential treatment was applied compared with the corresponding simultaneous combined treatment, highlighting for the first time the importance of treatment time schedule for LRAs combinations [9].

Here, we grouped the data from these two studies to investigate the role of the HIV-1 reservoir size in the reactivation capacity by LRAs in ex-vivo cultures of $\mathrm{CD}^{+}{ }^{+}$-depleted PBMCs isolated from 54 cART-treated patients and of resting $\mathrm{CD}^{+}{ }^{+} \mathrm{T}$ cells isolated from 30 cART-treated patients.

\section{Methods}

\section{Study participants}

We grouped the data from our two most recent reactivation studies to allow for an accurate and statistically relevant analysis of the results $[8,9]$. Indeed, the addition of our data from reactivation assays performed in these two studies allowed us to investigate the role of the HIV-1 reservoir size in the reactivation capacity by LRAs in three ex-vivo cellular models for HIV-1 postintegration latency:

(1) Ex-vivo patient cell cultures of 6 million $\mathrm{CD}^{+}{ }_{-}$ depleted PBMCs (at 2 million cells $/ \mathrm{ml}$ ) from 43 cARTtreated $\mathrm{HIV}^{+}$patients, in the absence of cART [24 patients (P1-P24) from [8] and 19 patients (P25-P43) from [9]].

(2) Ex-vivo patient cell cultures of 0.5 million resting $\mathrm{CD}^{+}{ }^{+} \mathrm{T}$ cells (at 0.8 million cells $/ \mathrm{ml}$ ) isolated from 30 cART-treated $\mathrm{HIV}^{+}$patients $[14$ patients (P44-P57) from [8] and 16 patients (P58-P73) from [9]].

(3) Ex-vivo patient cell cultures of 12 million $\mathrm{CD} 8^{+}$ depleted PBMCs (at 2 million cells $/ \mathrm{ml}$ ) isolated from 11 cART-treated $\mathrm{HIV}^{+}$patients, in the presence of cART antiretrovirals [efavirenz (4624, the AIDS Research and Reference Reagent Program, National Institutes of Health (NIH) Bethesda, Maryland, USA; $100 \mathrm{nmol} / \mathrm{l}$ ); zidovudine (3485, the AIDS Research and Reference Reagent Program, NIH; $180 \mathrm{nmol} / \mathrm{l})$; raltegravir (11680, the AIDS Research and Reference Reagent Program, NIH; 200 nmol/l)] (P74-P84) [8].

Characteristics of the patients for which we have previously isolated and stimulated, in the absence of cART, $\mathrm{CD} 8^{+}$-depleted $\mathrm{PBMCs}$ or resting $\mathrm{CD} 4^{+} \mathrm{T}$ cells are presented in Table 1. Characteristics of the patients for which we have previously isolated $\mathrm{CD} 8^{+}{ }_{-}$ depleted PBMCs and performed reactivation assays, in the presence of CART, are presented in Table 2 . Isolation and stimulation of $\mathrm{CD}^{+}{ }^{-}$-depleted PBMCs and of resting $\mathrm{CD} 4^{+} \mathrm{T}$ cells as well as quantification of viral RNA have been performed in the same lab for the whole patient cohort, as previously described [10]. Briefly, 1 day after isolation, cells were mock-treated or treated with anti-CD3 + anti-CD28 antibodies as a positive control or by various LRAs for 6 days. HIV-1 RNA levels in culture supernatants were next determined using the reverse transcription quantitative polymerase chain reaction (RT-qPCR)-based Generic HIV Charge Virale kit (Biocentric, Bandol, France). Cell cultures exhibiting reactivation level of supernatant HIV-1 RNA higher than 15, 150 or 200 copies/ $\mathrm{ml}$ (depending on the tested supernatant volumes) have been considered as positive measurements (Tables 1 and 2 ). We defined for each patient a frequency of positive measurements as the ratio between the number of 
Table 1. Characteristics of HIV-1-infected individuals from whom $\mathrm{CDB}^{+}$-depleted PBMCs were isolated, named P1-P43 and from whom resting $\mathrm{CD4}^{+} \mathrm{T}$ cells were isolated, named P44-P73.

\begin{tabular}{|c|c|c|c|c|c|c|c|c|c|c|c|c|c|c|c|c|c|}
\hline & Patients & $\begin{array}{l}\text { Year } \\
\text { of } \\
\text { birth }\end{array}$ & $\begin{array}{l}\text { Year } \\
\text { of } \\
\text { HIV } \\
\text { iagnostics }\end{array}$ & HIV & $\begin{array}{c}\mathrm{CD}^{+} \\
\text {nadir } \\
\text { (cells/ } \\
\mu \mathrm{l} \text { ) }\end{array}$ & $\begin{array}{c}\begin{array}{c}\text { Year } \\
\text { of }\end{array} \\
\text { first } \\
\text { treatment }\end{array}$ & $\begin{array}{c}\text { Year } \\
\text { of } \\
\text { undetectable } \\
\text { status }\end{array}$ & $\begin{array}{c}\text { Year } \\
\text { of } \\
\text { blood } \\
\text { sampling }\end{array}$ & $\begin{array}{c}\mathrm{CD}^{+} \\
\text {T-cell } \\
\text { count } \\
(\text { cells/ } \\
\mu \mathrm{l})\end{array}$ & Last treatment & $\begin{array}{c}\text { Year of } \\
\text { the } \\
\text { beginning } \\
\text { of } \\
\text { the } \\
\text { ongoing } \\
\text { treatment }\end{array}$ & $\begin{array}{c}\text { HIV } \\
\text { DNA } \\
\log _{10} \\
\text { copies/10 } 10^{6} \\
\text { cells }\end{array}$ & $\begin{array}{c}\text { HIV } \\
\text { DNA } \\
\text { copies } / 10^{6} \\
\text { cells }\end{array}$ & $\begin{array}{c}\text { Number } \\
\text { of } \\
\text { tested } \\
\text { conditions }\end{array}$ & $\begin{array}{c}\text { Number } \\
\text { of } \\
\text { reactivated } \\
\text { patient } \\
\text { cell } \\
s \text { cultures }\end{array}$ & $\begin{array}{l}\text { Frequency } \\
\text { of } \\
\text { HIV-1 } \\
\text { recovery } \\
(\%)\end{array}$ & $\begin{array}{c}\text { Median } \\
\text { level } \\
\text { of } \\
\text { HIV } \\
\text { RNA } \\
\text { copies/ } \\
\text { condition }\end{array}$ \\
\hline \multirow{43}{*}{$\begin{array}{l}\mathrm{CD}^{+} \\
\quad \text {-depleted } \\
\text { PBMCs }\end{array}$} & P1 & 1966 & 1994 & $x$ & 384 & 1997 & 1998 & 2013 & 905 & $\mathrm{ABC} / 3 \mathrm{TC} / \mathrm{ZDV}$ & 2004 & 2.833 & 680 & 16 & 9 & 56.3 & 300 \\
\hline & P2 & 1957 & 2004 & $x$ & 45 & 2004 & 2005 & 2013 & 351 & EFV/TDF/FTC & 2010 & 3.085 & 1217 & 16 & 1 & 6.3 & 5 \\
\hline & P3 & 1972 & 1994 & $\mathrm{X}$ & 138 & 1994 & 2002 & 2013 & 747 & TDF/FTC/FPV/r & 2010 & 1.845 & 70 & 16 & 11 & 68.8 & 584 \\
\hline & P4 & 1961 & 2007 & $\mathrm{X}$ & 364 & 2011 & 2011 & 2013 & 659 & $\mathrm{ABC} / 3 \mathrm{TC} / \mathrm{ATV} / \mathrm{r}$ & 2013 & 2.544 & 350 & 15 & 12 & 80.0 & 394 \\
\hline & P5 & 1971 & 2008 & $\mathrm{x}$ & 603 & 2008 & 2011 & 2013 & 758 & EFV/TDF/FTC & 2008 & 3.251 & 1782 & 16 & 12 & 75.0 & 3235 \\
\hline & P6 & 1971 & 2012 & $\mathrm{x}$ & 477 & 2012 & 2012 & 2013 & 607 & TDF/FTC/ATV/r & 2012 & 2.825 & 669 & 16 & 15 & 93.8 & 1792 \\
\hline & P7 & 1982 & 2008 & X & 620 & 2009 & 2009 & 2013 & 889 & TDF/FTC/RPV & 2009 & 2.826 & 670 & 14 & 9 & 64.3 & 378 \\
\hline & P8 & 1965 & 1995 & $05 \mathrm{DF}$ & 11 & 1995 & 2006 & 2013 & 990 & DRV/r/RAL & 2011 & 2.893 & 782 & 16 & 10 & 62.5 & 421 \\
\hline & P9 & 1939 & 2001 & X & 267 & 2001 & 2006 & 2013 & 1198 & EFV/ABC/3TC & 2006 & 2.928 & 847 & 16 & 7 & 43.8 & 51 \\
\hline & P10 & 1964 & 1998 & $x$ & 1 & 2005 & 2006 & 2013 & 506 & $\mathrm{TDF} / \mathrm{FTC} / \mathrm{FPV} / \mathrm{r}$ & 2009 & 2.538 & 345 & 16 & 6 & 37.5 & 0 \\
\hline & $\mathrm{P} 11$ & 1977 & 2008 & $02 \mathrm{AG}$ & 143 & 2009 & 2009 & 2013 & 494 & EFV/TDF/FTC & 2010 & 3.157 & 1435 & 16 & 12 & 75.0 & 984 \\
\hline & $\mathrm{P} 12$ & 1965 & 1996 & X & 11 & 1996 & 2010 & 2013 & 657 & TDF/FTC/NVP & 2012 & 2.976 & 947 & 16 & 7 & 43.8 & 0 \\
\hline & P13 & 1953 & 1995 & $x$ & 199 & 1995 & 2004 & 2013 & 847 & $\mathrm{ABC} / 3 \mathrm{TC} / \mathrm{SQV} / \mathrm{r}$ & 2007 & 2.403 & 253 & 16 & 5 & 31.3 & 0 \\
\hline & P14 & 1961 & 1996 & $x$ & 92 & 1996 & 2005 & 2013 & 770 & EFV/TDF/FTC & 2010 & 3.261 & 1823 & 16 & 9 & 56.3 & 189 \\
\hline & P15 & 1960 & 1995 & $x$ & 314 & 1997 & 2003 & 2013 & 633 & $\mathrm{ABC} / 3 \mathrm{TC} / \mathrm{FPV} / \mathrm{r}$ & 2012 & 2.591 & 390 & 16 & 6 & 37.5 & 1 \\
\hline & P16 & 1953 & 2006 & $x$ & 340 & 2006 & 2007 & 2013 & 641 & ABC/3TC/NVP & 2008 & 2.531 & 340 & 16 & 6 & 37.5 & 0 \\
\hline & P17 & 1973 & 1997 & $x$ & 395 & 1997 & 2010 & 2013 & 585 & EFV/TDF/FTC & 2010 & 2.420 & 263 & 16 & 6 & 37.5 & 0 \\
\hline & P18 & 1955 & 2003 & $x$ & 77 & 2012 & 2012 & 2013 & 313 & TDF/FTC/ATV/r & 2012 & 2.522 & 333 & 16 & 13 & 81.3 & 1033 \\
\hline & P19 & 1959 & 2009 & $x$ & 331 & 2009 & 2010 & 2013 & 815 & EFV/TDF/FTC & 2010 & 2.272 & 187 & 16 & 5 & 31.3 & 0 \\
\hline & P20 & 1965 & 2009 & $x$ & 316 & 2010 & 2010 & 2013 & 895 & TDF/FTC/ATV/r & 2011 & 3.092 & 1236 & 16 & 12 & 75.0 & 849 \\
\hline & P21 & 1968 & 1995 & $x$ & 185 & 1995 & 2000 & 2013 & 890 & TDF/FTC/NVP & 2009 & 2.778 & 600 & 17 & 8 & 47.1 & 11 \\
\hline & P22 & 1952 & 2005 & $x$ & 133 & 2005 & 2005 & 2013 & 319 & EFV/TDF/FTC & 2011 & 2.867 & 737 & 17 & 10 & 58.8 & 869 \\
\hline & P23 & 1964 & 1996 & $\mathrm{X}$ & 435 & 1996 & 2001 & 2013 & 531 & $\mathrm{ABC} / 3 \mathrm{TC} / \mathrm{ZDV}$ & 2002 & 2.580 & 380 & 16 & 10 & 62.5 & 1062 \\
\hline & P24 & 1963 & 1990 & $\mathrm{x}$ & 140 & 1990 & 2003 & 2014 & 752 & TDF/FTC/ATV/r & 2010 & 2.307 & 203 & 17 & 8 & 47.1 & 0 \\
\hline & P25 & 1950 & 1999 & $\mathrm{X}$ & 290 & 2000 & 2000 & 2014 & 948 & $\mathrm{ABC} / 3 \mathrm{TC} / \mathrm{ZDV}$ & 2002 & 1.041 & 11 & 17 & 1 & 5.9 & 0 \\
\hline & P26 & 1961 & 1997 & B & 224 & 1999 & 2009 & 2014 & 590 & TDF/FTC/ETR/RAL & 2011 & 2.826 & 670 & 13 & 4 & 30.8 & 0 \\
\hline & P27 & 1960 & 1997 & $x$ & 185 & 1997 & 2003 & 2014 & 428 & EFV/TDF/FTC & 2010 & 2.667 & 464 & 16 & 5 & 31.3 & 0 \\
\hline & P28 & 1963 & 2006 & $x$ & 256 & 2006 & 2006 & 2014 & 633 & TDF/FTC/ETR/RAL & 2009 & 2.769 & 587 & 17 & 8 & 47.1 & 80 \\
\hline & P29 & 1971 & 1997 & $\mathrm{D}$ & 255 & 1997 & 2009 & 2014 & 786 & $\begin{array}{c}\text { 3TC/ZDV/RAL/ } \\
\text { MVC }\end{array}$ & 2009 & 2.860 & 724 & 17 & 9 & 52.9 & 314 \\
\hline & P30 & 1969 & 2011 & F2 & 364 & 2012 & 2012 & 2014 & 723 & $\mathrm{ABC} / 3 \mathrm{TC} / \mathrm{ATV} / \mathrm{r}$ & 2012 & 3.407 & 2552 & 17 & 9 & 52.9 & 216 \\
\hline & P31 & 1969 & 1998 & $x$ & 138 & 1999 & 2012 & 2014 & 723 & $\mathrm{ABC} / 3 \mathrm{TC} / \mathrm{ATV} / \mathrm{r}$ & 2012 & 2.739 & 548 & 17 & 3 & 17.6 & 0 \\
\hline & P32 & 1973 & 2005 & $x$ & 71 & 2005 & 2005 & 2014 & 513 & $\mathrm{TDF} / \mathrm{FTC} / \mathrm{FPV} / \mathrm{r}$ & 2009 & 3.037 & 1089 & 13 & 4 & 30.8 & 0 \\
\hline & P33 & 1963 & 1996 & $x$ & 360 & 1996 & 1998 & 2014 & 777 & $\mathrm{ABC} / 3 \mathrm{TC} / \mathrm{ATV} / \mathrm{r}$ & 2014 & 2.097 & 125 & 13 & 4 & 30.8 & 0 \\
\hline & P34 & 1951 & 1990 & $x$ & 144 & 1991 & 1997 & 2014 & 629 & TDF/FTC/NVP & 2014 & 2.872 & 744 & 13 & 3 & 23.1 & 0 \\
\hline & P35 & 1976 & 2005 & $x$ & 422 & 2012 & 2012 & 2014 & 603 & TDF/FTC/ATV/r & 2014 & 0.699 & 5 & 15 & 1 & 6.7 & 0 \\
\hline & P36 & 1947 & 1998 & B & 151 & 1998 & 2006 & 2014 & 881 & $\mathrm{ATV} / \mathrm{r}$ & 2009 & 2.943 & 876 & 17 & 7 & 41.2 & 22 \\
\hline & P37 & 1959 & 2000 & B & 193 & 2000 & 2000 & 2014 & 547 & EFV/TDF/FTC & 2011 & 2.936 & 863 & 17 & 10 & 58.8 & 200 \\
\hline & P38 & 1972 & 2000 & $x$ & 146 & 2000 & 2002 & 2014 & 625 & TDF/FTC/NVP & 2009 & 1.756 & 57 & 15 & 2 & 13.3 & 0 \\
\hline & P39 & 1967 & 2006 & $x$ & 289 & 2006 & 2006 & 2014 & 1175 & FTC/RPV/TDF & 2011 & 2.410 & 257 & 17 & 4 & 23.5 & 0 \\
\hline & P40 & 1966 & 2007 & $x$ & 371 & 2010 & 2011 & 2014 & 739 & $\mathrm{TDF} / \mathrm{FTC} / \mathrm{ATV} / \mathrm{r}$ & 2011 & 3.195 & 1567 & 17 & 4 & 23.5 & 0 \\
\hline & P41 & 1968 & 2008 & $x$ & 192 & 2008 & 2008 & 2014 & 354 & EFV/TDF/FTC & 2010 & 2.959 & 909 & 13 & 8 & 61.5 & 229 \\
\hline & P42 & 1972 & 1996 & $x$ & 373 & 1997 & 2009 & 2014 & 1015 & EFV/TDF/FTC & 2010 & 3.652 & 4486 & 17 & 15 & 88.2 & 513 \\
\hline & P43 & 1980 & 2006 & $x$ & 230 & 2006 & 2006 & 2014 & 623 & $\mathrm{ABC} / 3 \mathrm{TC} / \mathrm{NVP}$ & 2012 & 3.035 & 1083 & 9 & 9 & 100 & 504 \\
\hline \multirow{28}{*}{$\begin{array}{l}\text { Resting } \mathrm{CD}^{+}{ }^{+} \\
\mathrm{T} \text { cells }\end{array}$} & P44 & 1949 & 1995 & $x$ & 399 & 1997 & 2007 & 2014 & 869 & EFV/TDF/FTC & 2011 & 3.079 & 1200 & 9 & 3 & 33.3 & 0 \\
\hline & P45 & 1966 & 1998 & $x$ & 558 & 1998 & 1999 & 2014 & 670 & $3 \mathrm{TC} / \mathrm{ZDV} / \mathrm{NVP}$ & 1999 & 3.072 & 1179 & 9 & 3 & 33.3 & 0 \\
\hline & P46 & 1973 & 2004 & $x$ & 746 & 2004 & 2010 & 2014 & 848 & $\mathrm{TDF} / \mathrm{FTC} / \mathrm{LPV} / \mathrm{r}$ & 2008 & 2.049 & 112 & 9 & 7 & 77.8 & 676 \\
\hline & P47 & 1966 & 2006 & $x$ & 215 & 2006 & 2007 & 2014 & 418 & EFV/TDF/FTC & 2009 & 3.645 & 4414 & 9 & 5 & 55.6 & 503 \\
\hline & P48 & 1960 & 1989 & $\mathrm{C}$ & 53 & 1999 & 1999 & 2014 & 401 & TDF/FTC/NVP & 2009 & 3.250 & 1777 & 9 & 3 & 33.3 & 0 \\
\hline & P49 & 1954 & 1984 & $x$ & 61 & 1996 & 2011 & 2014 & 856 & $3 T C / Z D V / N V P$ & 2002 & 3.718 & 5220 & 8 & 5 & 62.5 & 570 \\
\hline & P50 & 1973 & 2004 & $x$ & 294 & 2004 & 2004 & 2014 & 736 & $\mathrm{ABC} / 3 \mathrm{TC} / \mathrm{NVP}$ & 2007 & 2.960 & 911 & 9 & 2 & 22.2 & 0 \\
\hline & P51 & 1956 & 1985 & B & 92 & 1991 & 2005 & 2014 & 818 & $\mathrm{DRV} / \mathrm{r} / \mathrm{FTC} / \mathrm{MVC}$ & 2010 & 3.584 & 3841 & 8 & 5 & 62.5 & 454 \\
\hline & P52 & 1995 & 1998 & $x$ & 251 & 1998 & 2006 & 2014 & 663 & EFV/TDF/FTC & 2010 & 2.976 & 946 & 9 & 6 & 66.7 & 392 \\
\hline & P53 & 1969 & 2001 & $x$ & 192 & 2001 & 2004 & 2014 & 928 & $\mathrm{ABC} / 3 \mathrm{TC} / \mathrm{ZDV}$ & 2002 & 2.720 & 525 & 9 & 2 & 22.2 & 0 \\
\hline & P54 & 1948 & 2009 & $x$ & 354 & 2009 & 2009 & 2014 & 817 & $\mathrm{ABC} / 3 \mathrm{TC} / \mathrm{NVP}$ & 2013 & 3.183 & 1523 & 9 & 3 & 33.3 & 0 \\
\hline & P55 & 1952 & 2000 & $01 \mathrm{AE}$ & 424 & 2002 & 2007 & 2014 & 1091 & EFV/TDF/FTC & 2010 & 3.966 & 9248 & 9 & 6 & 66.7 & 453 \\
\hline & P56 & 1969 & 2011 & F2 & 364 & 2012 & 2013 & 2013 & 723 & $\mathrm{ABC} / 3 \mathrm{TC} / \mathrm{ATV} / \mathrm{r}$ & 2012 & 3.686 & 4849 & 9 & 6 & 66.7 & 412 \\
\hline & P57 & 1967 & 1992 & $x$ & 362 & 1994 & 2000 & 2014 & 845 & TDF/FTC/NVP & 2009 & 3.071 & 1177 & 9 & 6 & 66.7 & 218 \\
\hline & P58 & 1975 & 2004 & B & 292 & 2006 & 2006 & 2014 & 561 & EFV/TDF/FTC & 2006 & 2.880 & 758 & 9 & 3 & 33.3 & 0 \\
\hline & P59 & 1948 & 2009 & $x$ & 354 & 2009 & 2009 & 2014 & 817 & $\mathrm{ABC} / 3 \mathrm{TC} / \mathrm{NVP}$ & 2013 & 3.183 & 1523 & 7 & 2 & 28.6 & 0 \\
\hline & $\mathrm{P} 60$ & 1955 & 2000 & $x$ & 157 & 2000 & 2000 & 2014 & 579 & TDF/FTC/ATV/r & 2009 & 3.340 & 2186 & 5 & 4 & 80.0 & 472 \\
\hline & P61 & 1978 & 1996 & $x$ & 0 & 1996 & 2010 & 2014 & 493 & $\mathrm{TDF} / \mathrm{FTC} / \mathrm{LPV} / \mathrm{r}$ & 2009 & 3.671 & 4691 & 7 & 3 & 42.9 & 172 \\
\hline & $\mathrm{P} 62$ & 1967 & 1992 & $x$ & 362 & 1994 & 2000 & 2014 & 845 & TDF/FTC/NVP & 2009 & 3.071 & 1177 & 7 & 1 & 14.3 & 81 \\
\hline & $\mathrm{P} 63$ & 1975 & 2004 & B & 292 & 2006 & 2006 & 2014 & 561 & EFV/TDF/FTC & 2010 & 2.880 & 758 & 7 & 0 & 0.0 & 0 \\
\hline & P64 & 1959 & 2009 & B & 629 & 2009 & 2009 & 2014 & 994 & EFV/TDF/FTC & 2010 & 2.500 & 316 & 7 & 3 & 42.9 & 0 \\
\hline & P65 & 1970 & 1996 & B & 331 & 1998 & 2010 & 2014 & 1079 & $\mathrm{ABC} / 3 \mathrm{TC} / \mathrm{ATV} / \mathrm{r}$ & 2010 & 3.499 & 3157 & 7 & 4 & 57.1 & 200 \\
\hline & P66 & 1976 & 2004 & $x$ & 288 & 2007 & 2007 & 2014 & 819 & $\mathrm{DRV} / \mathrm{r}$ & 2014 & 2.634 & 431 & 6 & 2 & 33.3 & 0 \\
\hline & P67 & 1945 & 1988 & $\mathrm{~B}$ & 9 & 1997 & 2002 & 2014 & 491 & DRV/r/RAL & 2011 & 2.928 & 847 & 7 & 1 & 14.3 & 0 \\
\hline & P68 & 1965 & 2001 & $x$ & 182 & 2002 & 2002 & 2014 & 789 & EFV/TDF/FTC & 2012 & 3.539 & 3460 & 7 & 3 & 42.9 & 118 \\
\hline & P69 & 1969 & 2011 & F2 & 364 & 2012 & 2012 & 2014 & 723 & $\mathrm{ABC} / 3 \mathrm{TC} / \mathrm{ATV} / \mathrm{r}$ & 2012 & 3.686 & 4849 & 7 & 6 & 85.7 & 788 \\
\hline & P70 & 1945 & 2000 & $x$ & 141 & 2000 & 2000 & 2014 & 469 & EFV/TDF/FTC & 2010 & 3.627 & 4232 & 7 & 7 & 100.0 & 2390 \\
\hline & P71 & 1970 & 2005 & B & 291 & 2005 & 2012 & 2014 & 493 & TDF/FTC/ATV/r & 2011 & 3.509 & 3226 & 7 & 7 & 100.0 & 782 \\
\hline
\end{tabular}


Table 1 (continued)

\begin{tabular}{|c|c|c|c|c|c|c|c|c|c|c|c|c|c|c|c|c|}
\hline Patients & $\begin{array}{l}\text { Year } \\
\text { of } \\
\text { birth }\end{array}$ & $\begin{array}{c}\text { Year } \\
\text { of } \\
\text { HIV } \\
\text { diagnostic }\end{array}$ & $\begin{array}{c}\text { HIV } \\
\text { subtype }\end{array}$ & $\begin{array}{c}\mathrm{CD}^{+} \\
\text {nadir } \\
\text { (cells/ } \\
\mu \mathrm{l})\end{array}$ & $\begin{array}{c}\text { Year } \\
\text { of } \\
\text { first } \\
\text { treatment }\end{array}$ & $\begin{array}{c}\text { Year } \\
\text { of } \\
\text { undetectable } \\
\text { status }\end{array}$ & $\begin{array}{c}\text { Year } \\
\text { of } \\
\text { blood } \\
\text { sampling }\end{array}$ & $\begin{array}{c}\mathrm{CD}^{+} \\
\text {T-cell } \\
\text { count } \\
(\text { cells/ } \\
\mu \mathrm{l})\end{array}$ & Last treatment & $\begin{array}{l}\text { Year of } \\
\text { the } \\
\text { beginning } \\
\text { of } \\
\text { the } \\
\text { ongoing } \\
\text { treatment }\end{array}$ & $\begin{array}{c}\text { HIV } \\
\text { DNA } \\
\log _{10} \\
\text { copies/10 } \\
\text { cells }\end{array}$ & $\begin{array}{c}\text { HIV } \\
\text { DNA } \\
\text { copies } 110^{6} \\
\text { cells }\end{array}$ & $\begin{array}{c}\text { Number } \\
\text { of } \\
\text { tested } \\
\text { conditions }\end{array}$ & $\begin{array}{c}\text { Number } \\
\text { of } \\
\text { reactivated } \\
\text { patient } \\
\text { cell } \\
\text { cultures }\end{array}$ & $\begin{array}{l}\text { Frequency } \\
\text { of } \\
\text { HIV-1 } \\
\text { recovery } \\
(\%)\end{array}$ & $\begin{array}{c}\text { Median } \\
\text { level } \\
\text { of } \\
\text { HIV } \\
\text { RNA } \\
\text { copies/ } \\
\text { condition }\end{array}$ \\
\hline P72 & 1963 & 2005 & $x$ & 237 & 2005 & 2008 & 2014 & 414 & EFV/TDF/FTC & 2009 & 3.714 & 5177 & 7 & 6 & 85.7 & 627 \\
\hline P73 & 1970 & 2001 & Not-B & 264 & 2002 & 2007 & 2014 & 697 & $\mathrm{ABC} / 3 \mathrm{TC} / \mathrm{RAL}$ & 2012 & 3.492 & 3101 & 7 & 3 & 42.9 & 0 \\
\hline
\end{tabular}

Characteristics (age, $\mathrm{CD} 4^{+}{ }^{\mathrm{T}}$-cell count, $\mathrm{CD} 4^{+}$nadir, antiviral regimens, duration of therapy, duration with undetectable plasma HIV-1 RNA level and HIV-1 subtypes) of patients from the CHU St-Pierre Hospital (Brussels, Belgium) are presented. ' $X^{\prime}$ indicates not reported. The total HIV-DNA level, the number of reactivated patient cell cultures (genomic viral RNA in culture supernatants higher than 150 or $200 \mathrm{copies} / \mathrm{ml}$ depending on tested supernatant volumes), the number of tested conditions, the frequency of positive measurements expressed in percentage and the median level of HIV RNA in the supernatants of cell cultures are also indicated.

positive measurements observed following the diverse LRA treatments and the total number of tested conditions including the mock condition and the positive control (anti-CD3 + anti-CD28 antibodies). We also calculated a median level of extracellular HIV-1 RNA levels measured in supernatants following diverse LRAs, positive control and mock treatments for each patient (Tables 1 and 2).

Total HIV-1 DNA was quantified by the Generic HIV DNA cell kit based on a TaqMan PCR (Biocentric, Bandol, France) [11].

\section{Statistical analyses}

Spearman's coefficient $\left(r_{s}\right)$ and corresponding $P$ values were calculated two tailed and with a confidence interval of 95\%. Analyses were performed using Prism version 6.0 (GraphPad software, San Diego, California, USA). Statistical significance interpreted as $P$ value 0.05 or less.

\section{Results}

Reactivation capacity by latency-reversing agents in ex-vivo cultures of $\mathrm{CDB}^{+}$-depleted peripheral blood mononuclear cells positively correlates with total HIV-1 DNA reservoir size

We first studied the role of the HIV-1 reservoir size in the reactivation capacity by LRAs in ex-vivo cultures of CD $8^{+}$-depleted PBMCs isolated from 43 cART-treated patients, in the absence of cART in the cell cultures.

As shown in Fig. 1a, we observed a statistically relevant positive correlation between the reservoir size measured by total HIV-1 DNA and the frequency of positive HIV-1 recovery measurements in response to various LRAs, assessed with the Spearman correlation coefficient $\left(r_{s}=0.3730 ; \quad P=0.0138\right)$. We next examined the correlation between the reservoir size and the median level of extracellular HIV-1 RNA measured in the supernatant of tested conditions for each patient. We also observed a statistically relevant positive correlation $\left(r_{s}=0.4125 ; P=0.0060\right)$ (Fig. 1b).

Table 2. Characteristics of HIV-1-infected individuals from which $\mathrm{CDB}^{+}$-depleted PBMCs were isolated and stimulated in the presence of combination antiretroviral therapy, named P74-P84.

\begin{tabular}{|c|c|c|c|c|c|c|c|c|c|c|c|c|c|c|c|c|}
\hline Patients & $\begin{array}{c}\text { Year } \\
\text { of } \\
\text { birth }\end{array}$ & $\begin{array}{c}\text { Year } \\
\text { of } \\
\text { HIV } \\
\text { diagnostics }\end{array}$ & $\begin{array}{c}\text { HIV } \\
\text { subtype }\end{array}$ & $\begin{array}{l}\mathrm{CD}^{+} \\
\text {nadir } \\
(\text { cells/ } \mu \mathrm{l})\end{array}$ & $\begin{array}{c}\text { Year } \\
\text { of } \\
\text { first } \\
\text { treatment }\end{array}$ & $\begin{array}{c}\text { Year } \\
\text { of } \\
\text { undetectable } \\
\text { status }\end{array}$ & $\begin{array}{c}\text { Year } \\
\text { of } \\
\text { blood } \\
\text { sampling }\end{array}$ & $\begin{array}{c}\mathrm{CD}^{+} \\
\text {T-cell } \\
\text { count } \\
\text { (cells } / \mu \mathrm{l} \text { ) }\end{array}$ & $\begin{array}{c}\text { Last } \\
\text { treatment }\end{array}$ & $\begin{array}{l}\text { Year of the } \\
\text { beginning } \\
\text { of the } \\
\text { ongoing } \\
\text { treatment }\end{array}$ & $\begin{array}{c}\text { HIV } \\
\text { DNA } \\
\log _{10} \\
\text { copies/10 } \\
\text { PBMCs }\end{array}$ & $\begin{array}{c}\text { HIV DNA } \\
\text { copies/ } \\
10^{6} \\
\text { PBMCs }\end{array}$ & $\begin{array}{c}\text { Number } \\
\text { of } \\
\text { tested } \\
\text { conditions }\end{array}$ & $\begin{array}{c}\text { Number of } \\
\text { reactivated } \\
\text { patient } \\
\text { cell } \\
\text { cultures }\end{array}$ & $\begin{array}{c}\text { Frequency } \\
\text { of } \\
\text { HIV-1 } \\
\text { recovery } \\
(\%)\end{array}$ & $\begin{array}{c}\text { Median level } \\
\text { of HIV } \\
\text { RNA } \\
\text { copies/ } \\
\text { condition }\end{array}$ \\
\hline P74 & 1967 & 2002 & $x$ & 482 & 2002 & 2009 & 2015 & 534 & FTC/TDF/NVP & 2009 & 3.243 & 1750 & 5 & 5 & 100.0 & 1053 \\
\hline P75 & 1952 & 1998 & $x$ & 316 & 1998 & 1999 & 2015 & 832 & FTC/TDF/NVP & 2009 & 2.580 & 380 & 5 & 4 & 80.0 & 75 \\
\hline P76 & 1944 & 1991 & $x$ & 15 & 1991 & 2002 & 2015 & 859 & $\mathrm{ATV} / \mathrm{r} / \mathrm{ABC} / 3 \mathrm{TC}$ & 2008 & 3.004 & 1010 & 5 & 5 & 100.0 & 63 \\
\hline P77 & 1969 & 1996 & $x$ & 200 & 1996 & 1997 & 2015 & 554 & $\mathrm{ABC} / 3 \mathrm{TC} / \mathrm{EFV}$ & 2006 & 2.646 & 443 & 5 & 5 & 100.0 & 56 \\
\hline P78 & 1966 & 1994 & $\mathrm{X}$ & 384 & 1997 & 2011 & 2015 & 778 & EFV/ATV/r & 2011 & 2.958 & 907 & 7 & 7 & 100.0 & 769 \\
\hline P79 & 1960 & 1993 & B & 187 & 1996 & 2011 & 2015 & 830 & LPV/r/NVP & 2010 & 2.926 & 843 & 7 & 7 & 100.0 & 257 \\
\hline P80 & 1939 & 1994 & $x$ & 272 & 1994 & 2002 & 2015 & 880 & 3TC/ZDV/EFV & 2002 & 3.430 & 2693 & 5 & 5 & 100.0 & 762 \\
\hline P81 & 1975 & 2011 & $x$ & 10 & 2011 & 2012 & 2015 & 453 & DRV/3TC/ZDV & 2012 & 2.859 & 723 & 7 & 5 & 71.4 & 24 \\
\hline P82 & 1967 & 1990 & B & 587 & 1990 & 2001 & 2015 & 2112 & $\mathrm{ABC} / 3 \mathrm{TC} / \mathrm{ZDV}$ & 2002 & 2.919 & 830 & 7 & 4 & 57.1 & 15 \\
\hline P83 & 1990 & 2013 & $x$ & 515 & 2013 & 2014 & 2015 & 1400 & DTG/ABC/3TC & 2013 & 3.074 & 1187 & 7 & 7 & 100.0 & 656 \\
\hline P84 & 1979 & 2008 & B & 135 & 2009 & 2009 & 2015 & 463 & $\mathrm{EFV} / \mathrm{c} / \mathrm{TDF} / \mathrm{FTC}$ & 2014 & 2.831 & 677 & 7 & 7 & 100.0 & 227 \\
\hline
\end{tabular}

Characteristics (age, $\mathrm{CD} 4^{+} \mathrm{T}$-cell count, $\mathrm{CD} 4^{+}$nadir, antiviral regimens, duration of therapy, duration with undetectable plasma HIV-1 RNA level, and HIV-1 subtypes) of patients from the CHU St-Pierre Hospital (Brussels, Belgium) are presented. ' $X$ ' indicates not reported. The total HIV-DNA level, the number of reactivated patient cell cultures (genomic viral RNA in culture supernatants higher than $15 \mathrm{copies} / \mathrm{ml}$ ), the number of tested conditions, the frequency of positive measurements expressed in percentage and the median level of HIV RNA in the supernatants of cell cultures are also indicated. 
(a)

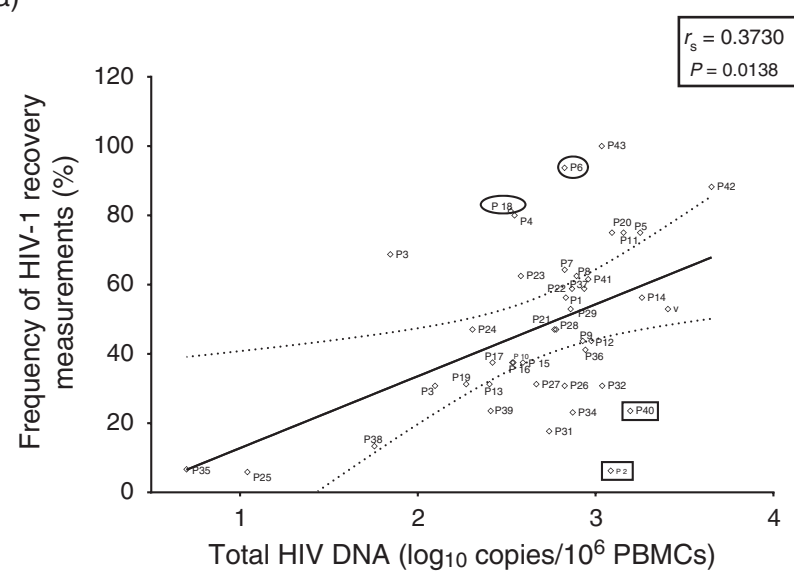

(c)

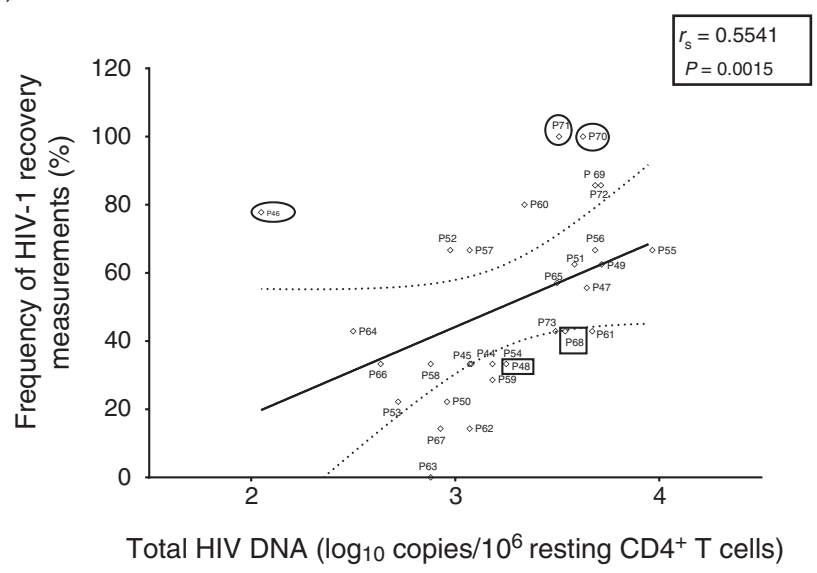

(b)

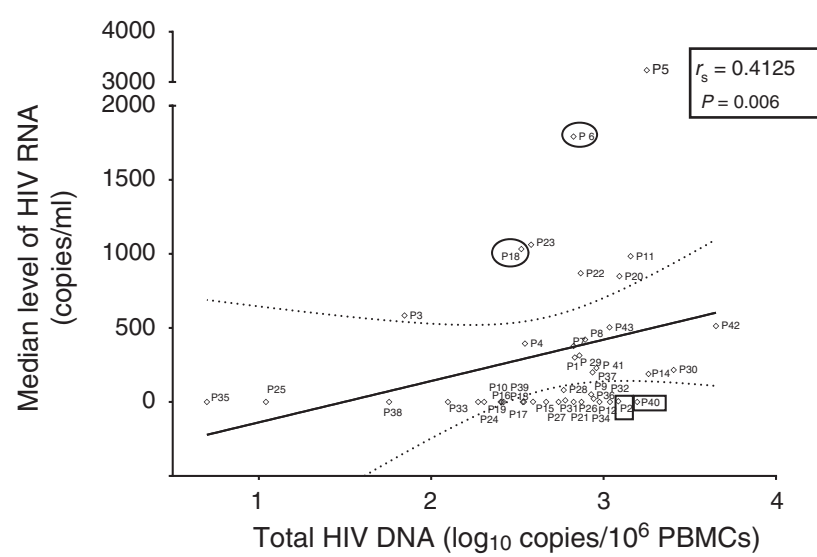

(d)

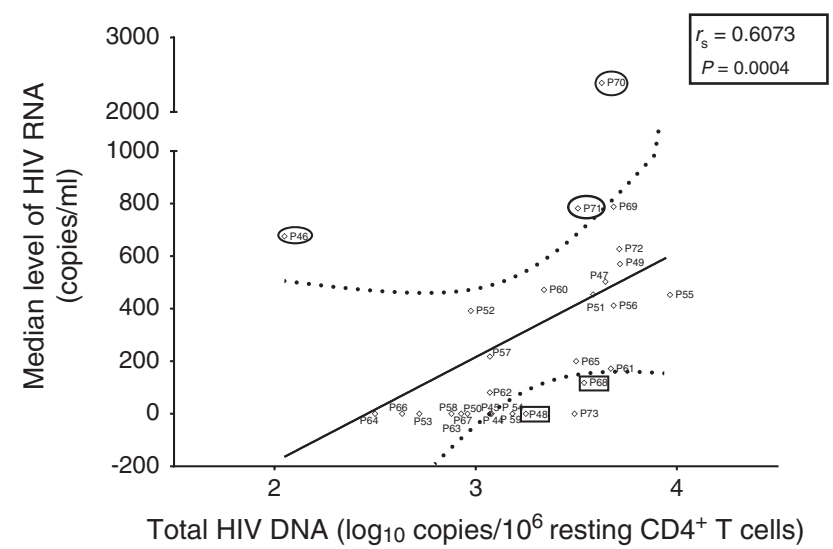

Fig. 1. Representation of the frequency of positive measurements observed in ex-vivo cultures and the median level of HIV RNA (copies $/ \mathrm{ml}$ ) in the supernatants of cell cultures following LRAs, positive control and mock treatments related to total HIV DNA. (a) Representation of the frequency of positive measurements observed in ex-vivo cultures of CD8 ${ }^{+}$-depleted PBMCs following latency-reversing agents, positive control and mock treatments related to total HIV DNA (43 patients from Table 1, P1-P43). We observed a statistically relevant correlation as indicated. We also identified patients with high capacities of cell reactivation despite low level of total HIV-DNA (e.g. P6 and P18, indicated by circles), whereas others presented low cell reactivation capacities relative to their high total HIV-DNA level (e.g. P2 and P40, indicated by rectangles). (b) Representation of the median level of HIV RNA (copies/ml) in the supernatants of cell cultures of CD8 ${ }^{+}$-depleted PBMCs following latency-reversing agents, positive control and mock treatments related to total HIV DNA. The analysis was performed on cultures described in (a) (P1 -P43). We observed a statistically relevant correlation as indicated. Patients emphasized in (a) are highlighted in the same way. (c)

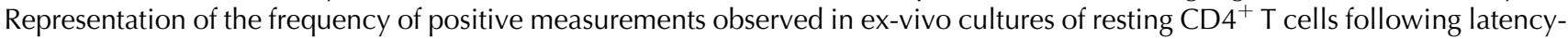
reversing agents, positive control and mock treatments related to total HIV DNA (30 patients from Table 1, P44-P73). We observed a statistically relevant correlation. We also identified patients with high capacities of cell reactivation despite low level of total HIV-DNA (e.g. P46, P70 and P71, indicated by circles), whereas others presented low cell reactivation capacities relative to their high total HIV-DNA level (e.g. P68 and P48 indicated by rectangles). (d) Representation of the median level of HIV RNA (copies $/ \mathrm{ml}$ ) in the supernatants of cell cultures of resting $\mathrm{CD} 4^{+} \mathrm{T}$ cells following latency-reversing agents, positive control and mock treatments related to total HIV DNA. The analysis was performed on cultures described in (c) (P44-P73). We observed a statistically relevant correlation as indicated. Patients emphasized in (c) are highlighted in the same way.

\section{Reactivation capacity by latency-reversing agents in ex-vivo cultures of resting $\mathrm{CD4}^{+} \mathrm{T}$ cells positively correlates with total HIV-1 DNA reservoir size}

We confirmed the positive correlations that we observed in $\mathrm{CD}^{+}{ }^{+}$-depleted PBMCs between the HIV-1 reservoir size and either the frequency or the level of reactivation in ex-vivo cultures of resting $\mathrm{CD}^{+}{ }^{+} \mathrm{T}$ cells isolated from 30 cART-treated $\mathrm{HIV}^{+}$aviremic patients (Fig. 1c and d, respectively). In this case, the correlations were even statistically stronger $\left(r_{s}=0.5541 ; P=0.0015\right.$ and $r_{s}=0.6073 ; P=0.0004$, respectively) than the correlations we observed in $\mathrm{CD}^{+}$-depleted PBMCs $\left(r_{s}=0.3730\right.$; $P=0.0138$ and $r_{s}=0.4125 ; P=0.0060$, respectively). 
Altogether, our data strongly established positive correlations between the HIV-1 reservoir size and the ex-vivo capacity of HIV-infected patient cell cultures to be reactivated in response to different classes of LRAs.

However, the correlations were far from perfect correlations. Indeed, we identified $\mathrm{HIV}^{+}$patients whose frequency of positive measurements and median level of extracellular HIV-1 RNA deviated from linearity relative to their corresponding HIV reservoir size. For example, patients P6 and P18 exhibited a high frequency of positive measurements and a high median level of HIV-1 RNA relative to their corresponding HIV-1 DNA level (circles in Fig. 1a and b, respectively). In contrast, patients P2 and P40 exhibited opposite reactivation capacity relative to their corresponding HIV-1 DNA level (rectangles in Fig. 1a and b). We observed similar data in the resting CD4 ${ }^{+}$T-cell population for patients $\mathrm{P} 46, \mathrm{P} 70$ and $\mathrm{P} 71$ (Fig. 1c and d, circles) and for patients $\mathrm{P} 48$ and P68 (Fig. 1c and d, rectangles).

\section{Median level of extracellular HIV-1 RNA after treatment by latency-reversing agents in ex-vivo cultures of $\mathrm{CDB}^{+}$-depleted peripheral blood mononuclear cells in the presence of combination antiretroviral therapy positively correlates with total HIV-1 DNA reservoir size}

The fact that some patients escape from the correlations could be explained by the fact that in addition to the size of the HIV-1 reservoir, additional elements could be involved in the reactivation capacity of patient cell cultures, such as variations in the strength of HIV-1 transcriptional repression but also different amplifications of newly produced viruses in cell cultures due to variable capacities of cells to get reinfected by HIV-1 (an effect which is possibly LRA-induced) or to different viral fitness. Therefore, we took advantage of our previous published reactivation experiments in $\mathrm{CD} 8^{+}$-depleted PBMCs isolated from 11 aviremic patients (Table 2), performed in the presence of CART to prevent de novo infection by newly produced virions [8], to explore the correlation between the median level of extracellular HIV-1 RNA following LRA treatments and the reservoir size. The correlation between the reservoir size and the frequency of positive HIV-1 recovery measurements was not analyzed as the sensitivity of the HIV-1 RNA quantification in these assays in the presence of cART allowed us to detect very low levels of viral production in almost all the cell cultures.

Interestingly, we observed again a statistically relevant positive correlation $\left(r_{s}=0.6455 ; P=0.0368\right)$ (Fig. 2). Moreover, this correlation was even stronger than the one observed in the absence of cART. Nevertheless, in these assays in the presence of cART, we also identified patients with high or low capacities of cell reactivation relative to their corresponding level of total HIV-1 DNA (P78

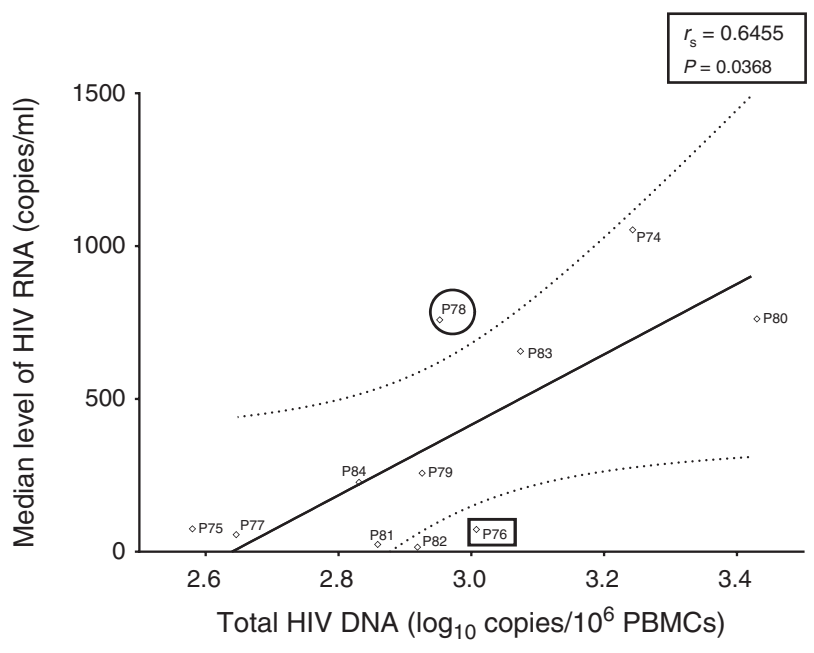

Fig. 2. Representation of the median level of HIV RNA (copies/ml) in the supernatants of $\mathrm{CD8}^{+}$-depleted PBMCs following latency-reversing agents, positive control and mock treatments in the presence of combination antiretroviral therapy related to total HIV DNA (11 patients from Table 2, P74-P84). We observed a statistically relevant correlation as indicated. We identified patients with high or low capacity of cell reactivation relative to their level of total HIV-DNA (P78 indicated by a circle and P76 indicated by a rectangle, respectively).

indicated by a circle and P76 indicated by a rectangle, respectively, in Fig. 2).

Consequently, we demonstrated a positive correlation between the HIV-1 reservoir size and the ex-vivo capacity of HIV-infected patient cell cultures to be reactivated in response to different classes of LRAs in the three ex-vivo postintegration latency models evaluated in the present study. However, in these three models, we identified patients who escape from the correlation. The comparison of the results observed in the presence and in the absence of cART supported that these patient-specific variations were likely due to differences in the strength of HIV-1 transcriptional repression and not to different amplifications of newly produced viruses.

Ex-vivo reactivation assays performed in $\mathrm{CD8}^{+}$. depleted peripheral blood mononuclear cells allow the isolation of a greater number of latently infected cells compared with the ex-vivo assay performed in resting $\mathrm{CD4}^{+} \mathrm{T}$ cells

The small numbers of latently infected cells found in vivo hinders reactivation studies and forces researchers to withdraw great volumes of fresh whole blood from aviremic patients to perform ex-vivo assays. In the two studies $[8,9]$ retrospectively analyzed in this report, we isolated either $\mathrm{CD}^{+}{ }^{-}$-depleted PBMCs or resting CD $4^{+}$ $\mathrm{T}$ cells from $120 \mathrm{ml}$ of blood of cART-treated $\mathrm{HIV}^{+}$ patients. Our reactivation experiments were designed in such a way that we first performed the reactivation assays 
in $\mathrm{CD}^{+}{ }^{+}$-depleted PBMCs and selected the most potent and promising LRAs and combinations of LRAs for the ex-vivo reactivation assays performed in resting $\mathrm{CD}^{+}{ }^{-}$T-cell cultures.

Here, we calculated the median frequency of HIV-1 recovery (median number of the frequency of HIV-1 recovery calculated for each patient and expressed in percentage) in both ex-vivo assays, and we surprisingly found that the percentage of median frequency of HIV-1 recovery was similar in $\mathrm{CD} 8^{+}$-depleted PBMCs (47\%) and in resting $\mathrm{CD}^{+}{ }^{+} \mathrm{T}$ cells $(43 \%)$ despite the fact that, in the latter cell type, we tested the most potent LRAs and combinations of LRAs (Table 3 ) and we would therefore expect to reach higher median frequency of HIV-1 recovery. This observation of similar median values obtained in both cell types is due, at least partially, to the higher number of infected cells seeded in the CD8 ${ }^{+}$ depleted PBMCs ex-vivo assays (Table 3). Indeed, the median number of seeded HIV-1 copies per tested condition, obtained by the multiplication of total HIV-1 DNA copies $/ 10^{6}$ cells $\left(\mathrm{CD} 8^{+}\right.$-depleted PBMCs or resting $\mathrm{CD} 4^{+} \mathrm{T}$ cells) and of the number of plated cells per tested condition, was 4.9 times higher in cultures of $\mathrm{CD}^{+}$-depleted PBMCs than in cultures of resting $\mathrm{CD} 4^{+}$ $\mathrm{T}$ cells (Table 3 ).

In addition, the seeding of $\mathrm{CD} 8^{+}$-depleted PBMCs in medium provided a more physiological culture environment. Indeed, the production of cytokines or growth factors by cell types not present in cultures of resting $\mathrm{CD}^{+} \mathrm{T}$ cells could be another possible explanation for the higher frequency of HIV recovery in the $\mathrm{CD} 8{ }^{+}$-depleted PBMCs ex-vivo cultures compared with the resting $\mathrm{CD}^{+}{ }^{+} \mathrm{T}-$ cell ex-vivo cultures.

Finally, the median number of tested conditions was higher in the $\mathrm{CD} 8^{+}$-depleted PBMCs ex-vivo assays as the number of isolated cells was much higher (Table 3 ). This constitutes another advantage for the use of $\mathrm{CD} 8^{+}$ depleted PBMCs instead of resting $\mathrm{CD}^{+} \mathrm{T}$ cells as ex-vivo HIV-1 latency model.

In conclusion, in addition to providing a more physiological culture environment, the ex-vivo assays performed with $\mathrm{CD} 8^{+}$-depleted PBMCs allowed us to test a higher number of conditions containing a larger amount of proviruses. Therefore, this model should probably be favored in ex-vivo experiments when many conditions have to be tested.

\section{Discussion}

In this report, we grouped and retrospectively analyzed the data from our two very recent HIV-1 reactivation studies $[8,9]$ to assess the role of the HIV-1 reservoir size in the reactivation capacity by LRAs in ex-vivo cultures

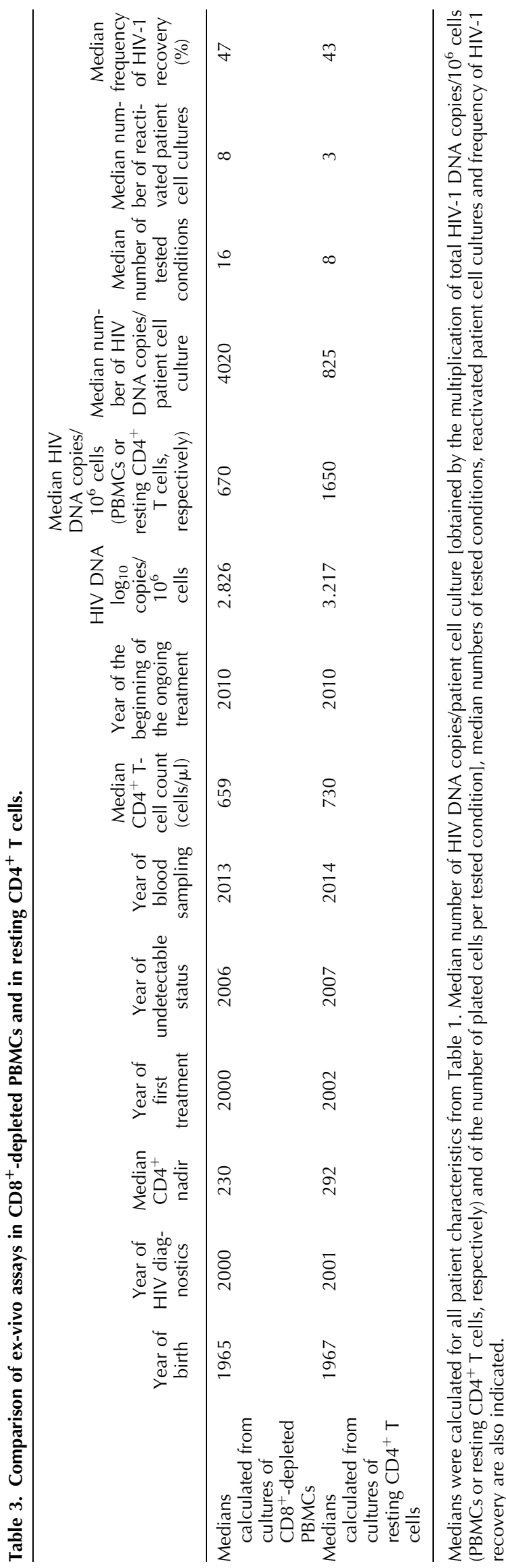


of $\mathrm{CD}^{+}{ }^{+}$-depleted PBMCs and of resting $\mathrm{CD} 4^{+} \mathrm{T}$ cells isolated from cART-treated patients.

We measured the HIV-1 reservoir size using a qPCRbased method for proviral DNA rather than culture-based assays. Indeed, each of these assays has its specific advantages and drawbacks [12]. Culture-based assays and PCR-based assays, respectively, underestimate and overestimate the size of HIV-1 reservoir [12,13]. Even if these assays have their limitations, Kiselinova et al. [14] have recently reported important correlations between the viral outgrowth assay and total HIV-1 DNA measures, demonstrating that the total pool of HIV-1 DNA predicts the size of the replication-competent virus in cART-suppressed patients. In addition, multiple recent treatment interruption trials have revealed a correlation between total HIV-1 DNA levels and time to viral rebound following cART interruption $[15,16]$. Altogether, these studies indicate that PCR-based assays provide an accurate and reproducible estimate of the HIV-1 reservoir size.

We strongly established a statistically significant positive correlation between the size of the HIV-1 reservoir and the frequency of positive HIV-1 recovery measurements in response to various LRAs in ex-vivo cultures of $\mathrm{CD} 8^{+}-$ depleted PBMCs. We next demonstrated a statistically relevant positive correlation between reservoir size and the median level of extracellular HIV RNA measured in the supernatants of tested conditions for each patient and confirmed these two correlations in ex-vivo cultures of resting $\mathrm{CD} 4^{+} \mathrm{T}$ cells. To the best of our knowledge, this report constitutes the first demonstration of a strong and statistically relevant positive correlation between the reservoir size and the reactivation capacity by LRAs $e x$ vivo, relying on a very large amount of patients $(n=84)$.

However, the correlations were far from perfect correlations. Indeed, we identified $\mathrm{HIV}^{+}$patients whose frequency of positive measurements and median level of extracellular HIV-1 RNA deviated from linearity relative to their corresponding HIV reservoir size. We obtained similar results in the presence and in the absence of cART, supporting the notion that the heterogeneity observed between patients was not due to different viral amplifications in cell cultures.

The possibility that the outliers could be due to alterations in primer/probe binding sites is highly unlikely. Indeed, the HIV DNA Biocentric kit we used is based on a TaqMan PCR with primers and internal probe targeting a conserved consensus region in the long terminal repeat of the HIV-1 major group. These primers and probe have proved their ability to quantify the HIV-1 genome of different clades in large cohorts of $\mathrm{HIV}^{+}$patients from various resource-limited countries in which several HIV-1 subtypes or circulating recombinant forms are present [17].
Consequently, the heterogeneity that we observed between patients in terms of reactivation capacity of their ex-vivo cell cultures indicates that patients with similar reservoir sizes assessed by total HIV-1 DNA may have differences in the strength of HIV-1 transcriptional repression resulting from the establishment of different molecular mechanisms of viral persistence. For instance, these mechanisms might vary depending on the T-cell subsets, which have been found to be impacted by patient history [18-20]. In this regard, we can imagine that not only the patient characteristics (e.g. genetic background, time to treatment initiation, duration and type of therapy) but also viral specificities and virus-host interaction features may have a significant role in the establishment and the maintenance of the latent reservoirs and their capacity to produce viruses, leading, at the molecular level, to the establishment of a multitude of mechanisms that regulate latency and probably vary from one patient to the other and even from one cell to the other in a single patient.

The patient-to-patient diversity that we observed and which constitutes a major finding of this report raises the possibility that a molecular-based or clinical-based individualized LRA treatment could be more efficient than a nonpersonalized treatment, even if this later treatment is potent ex-vivo. It emphasizes the need to evaluate the efficacy of an LRA first ex vivo in cell cultures from a given patient before the administration of this LRA to this given patient in vivo in the context of a clinical trial. The efficacy of an LRA in a given patient cannot be generalized to a cohort of patients. In the vast majority of clinical trials aimed at reactivating HIV-1 from latency, a preselection based on HIV-1 DNA reservoir size and on the ex-vivo reactivation assays has not been performed. It might partially explain the failure of these clinical trials. The possibility that the molecular mechanisms of latency vary even from one cell to the other in a single patient also explains that the extent of latency reversal currently achieved in clinical trials using an LRA targeting a single mechanism of viral persistence is insufficient to mobilize significant proportions of the latent reservoir.

In conclusion, the reservoir size is one predictive marker of LRA effectiveness but this parameter alone is not sufficient. The identification of other predictive markers is necessary to predict the success of HIV antilatency approaches. This could allow the selection of the $\mathrm{HIV}^{+}$ cART-treated patients who could respond to 'shock' strategies.

In this report, we were not able to identify patient characteristics implicated in the patient-specific reactivation variations. Therefore, well designed prospective studies aimed at understanding the potential determinants involved in this interpatient variability are absolutely needed and could lead to identification of key elements in 
the race for HIV durable remission or hopefully for HIV cure.

\section{Acknowledgements}

We thank Ludivine David and Adeline Melard from Christine Rouzioux's laboratory for excellent technical assistance. We thank Karine Fombellida from ULg for her precious advices. G.D., C.R. and C.V.L. wrote the article. G.D., S.B., A.K., B.V.D., N.D., C.V., V.A.F., N.C., S.D.W., O.R., C.R. and C.V.L. participated to the data analyses.

This work was supported by the ANRS (France Recherche Nord\&Sud Sida-HIV Hépatites), the Belgian Fund for Scientific Research (FRS-FNRS, Belgium), the 'Fondation Roi Baudouin', the NEAT program, the Walloon Region (the Excellence Program 'Cibles') and the International Brachet Stiftung (IBS). S.B. is a fellow of the Belgian «Fonds pour la Recherche dans l'Industrie et l'Agriculture» (FRIA). A.K. is a postdoctoral fellow of 'Les Amis des Instituts Pasteur' à Bruxelles, asbl. B.V.D. is a postdoctoral fellow from the ANRS. N.D. is supported by a "PDR" grant from the FRS-FNRS. G.D. and C.V.L. are 'Aspirant' and 'Directeur de Recherches' of the FRSFNRS (Belgium), respectively.

\section{Conflicts of interest}

There are no conflicts of interest.

\section{References}

1. Colin L, Van Lint C. Molecular control of HIV-1 postintegration latency: implications for the development of new therapeutic strategies. Retrovirology 2009; 6:111.

2. Richman DD, Margolis DM, Delaney M, Greene WC, Hazuda $D$, Pomerantz RJ. The challenge of finding a cure for HIV infection. Science 2009; 323:1304-1307.

3. Shan L, Deng K, Shroff NS, Durand CM, Rabi SA, Yang HC, et al. Stimulation of HIV-1-specific cytolytic T lymphocytes facilitates elimination of latent viral reservoir after virus reactivation. Immunity 2012; 36:491-501.

4. Van Lint C, Bouchat S, Marcello A. HIV-1 transcription and latency: an update. Retrovirology 2013; 10:67.

5. Jiang G, Mendes EA, Kaiser P, Wong DP, Tang Y, Cai I, et al. Synergistic reactivation of latent HIV expression by ingenol-3angelate, PEP005, targeted NF-kB signaling in combination with JQ1 induced p-TEFb activation. PLOS Pathog 2015; 11:e1005066.

6. Reuse S, Calao M, Kabeya K, Guiguen A, Gatot JS, Quivy V, et al. Synergistic activation of HIV-1 expression by deacetylase inhibitors and prostratin: implications for treatment of latent infection. PLoS One 2009; 4:e6093.
7. Laird GM, Bullen CK, Rosenbloom DI, Martin AR, Hill AL, Durand $C M$, et al. Ex vivo analysis identifies effective HIV-1 latency-reversing drug combinations. J Clin Invest 2015; 125:1901-1912.

8. Darcis G, Kula A, Bouchat S, Fujinaga K, Corazza F, Ait-Ammar $A$, et al. An in-depth comparison of latency-reversing agent combinations in various in vitro and ex vivo HIV-1 latency models identified bryostatin-1+JQ1 and ingenol-B+JQ1 to potently reactivate viral gene expression. PLOS Pathog 2015; 11:e1005063.

9. Bouchat S, Delacourt N, Kula A, Darcis G, Van Driessche B, Corazza $F$, et al. Sequential treatment with 5-aza-2'-deoxycytidine and deacetylase inhibitors reactivates HIV-1. EMBO Mol Med 2015; 8:117-138.

10. Bouchat S, Gatot JS, Kabeya K, Cardona C, Colin L, Herbein G, et al. Histone methyltransferase inhibitors induce HIV-1 recovery in resting CD4+ T cells from HIV-1+ HAART-treated patients. AIDS 2012; 26:1473-1482.

11. Avettand-Fenoel V, Chaix ML, Blanche S, Burgard M, Floch C, Toure K, et al. LTR real-time PCR for HIV-1 DNA quantitation in blood cells for early diagnosis in infants born to seropositive mothers treated in HAART area (ANRS CO 01). I Med Virol 2009; 81:217-223.

12. Bruner KM, Hosmane NN, Siliciano RF. Towards an HIV-1 cure: measuring the latent reservoir. Trends Microbiol 2015; 23:192-203.

13. Ho YC, Shan L, Hosmane NN, Wang J, Laskey SB, Rosenbloom $\mathrm{DI}$, et al. Replication-competent noninduced proviruses in the latent reservoir increase barrier to HIV-1 cure. Cell 2013; 155:540-551.

14. Kiselinova $M$, De Spiegelaere $W$, Buzon MJ, Malatinkova $E_{,}$ Lichterfeld M, Vandekerckhove L. Integrated and total HIV-1 DNA predict ex vivo viral outgrowth. PLOS Pathog 2016; 12:e1005472.

15. Azzoni L, Foulkes AS, Papasavvas E, Mexas AM, Lynn KM, Mounzer K, et al. Pegylated Interferon alfa-2a monotherapy results in suppression of HIV type 1 replication and decreased cell-associated HIV DNA integration. / Infect Dis 2013; 207:213-222.

16. Williams JP, Hurst J, Stohr W, Robinson N, Brown H, Fisher M, et al. HIV-1 DNA predicts disease progression and posttreatment virological control. Elife 2014; 3:e3821.

17. Rouet F, Chaix ML, Nerrienet E, Ngo-Giang-Huong N, Plantier JC, Burgard $M$, et al. Impact of HIV-1 genetic diversity on plasma HIV-1 RNA Quantification: usefulness of the Agence Nationale de Recherches sur le SIDA second-generation long terminal repeat-based real-time reverse transcriptase polymerase chain reaction test. I Acquir Immune Defic Syndr 2007; 45:380-388.

18. Cheret A, Bacchus-Souffan C, Avettand-Fenoel V, Melard A, Nembot G, Blanc C, et al. Combined ART started during acute HIV infection protects central memory CD4+ T cells and can induce remission. I Antimicrob Chemother 2015; 70:21082120.

19. Chomont N, El-Far M, Ancuta P, Trautmann L, Procopio FA, Yassine-Diab $B$, et al. HIV reservoir size and persistence are driven by $\mathbf{T}$ cell survival and homeostatic proliferation. Nat Med 2009; 15:893-900.

20. Descours B, Avettand-Fenoel V, Blanc C, Samri A, Melard A, Supervie $V$, et al. Immune responses driven by protective human leukocyte antigen alleles from long-term nonprogressors are associated with low HIV reservoir in central memory CD4 T cells. Clin Infect Dis 2012; 54: 1495-1503. 